\title{
Outcomes Research, Pharmacoeconomics and the Pharmaceutical Industry
}

Diana I. Brixner

\section{OBJECTIVE:}

To review the basic principles behind outcomes research and pharmacoeconomics within the pharmaceutical industry.

\section{DATA SOURCES:}

Author's knowledge and recent pharmacy literature.

\section{STUDY SELECTION:}

Not applicable.

\section{DATA EXTRACTION}

Not applicable.

\section{DATA SYNTHESIS:}

Various market forces have created the

\begin{abstract}
need for pharmacoeconomics research. The pharmaceutical industry has responded with the development of entire departments, interdisciplinary teams, or, at least, functional responsibility within a specific position to meet this need. As is critical to any new business, the customers of this information must be defined. With this knowledge in hand, pharmacists can design and implement outcomes research and pharmacoeconomic studies to meet the specific needs of a targeted health system. The interdisciplinary nature of this field requires an organization structured to meet the diverse methodologic approaches used in these studies.
\end{abstract}

\section{CONCLUSION:}

Armed with outcomes and pharmacoeconomic information, the pharmaceutical industry is far better situated to meet the challenge of integrating the use of pharmaceuticals into current practice.

\section{KEY WORDS:}

Pharmacy benefit management, Pharmacoeconomics, Industry, Managed care organizations, FDA, Outcomes research, Cost of care.

J Managed Care Pharm 1996; 2: 48-52.
Various market influences are driving the emphasis on costs and quality of health care delivery in the U.S. The health care budget has received substantial attention over the last several years, and the increased rate of health care spending must be brought under control.

In this article, I review basic concepts of pharmacoeconomics and its relation to outcomes research. The responses of the pharmaceutical industry to the need for such information is also explored.

\section{WHY DO PHARMACOECONOMICS?}

Employers have recognized that the cost of health care benefits can have a dramatic impact on their bottom line. These powerful forces have driven managed care plans to provide the employer with new options for health care at equal, if not better, quality at a lower cost. The initial reaction to this pressure was to negotiate discounts from hospital contractors, laboratory suppliers, and the pharmaceutical industry.

This scenario spawned the pharmacy benefit management company (PBM), which has been able to provide both managed care plans and employers with pharmaceutical products and services at guaranteed lower costs. The success of this short-term approach is evidenced by the significant drop in pressure felt by employee health care benefit managers over the last two years to reduce costs. ${ }^{1}$ However, once the ability to negotiate and provide lower costs has hit the minimum possible cost, the managed care and pharmaceutical industries must find new avenues to provide quality health care at lower costs.

Simultaneously, the consumers of health care are becoming more educated. Patients, employers, and payers want to know what they are getting for their health care dollar. If the value of pharmaceuticals is not demonstrated within this environment, then medications will be de-emphasized in health care budgets.

The practice of pharmacoeconomics and outcomes re-

\section{A Author \\ DIANA I. BRIXNER, R.P $H$., PH.D., is Director of Pharmaceutical Outcomes Research, Ciba Pharmaceuticals, Summit, NJ.}

CE CREDrr: This article is Continuing Education \# 692-233-96-002 and affords 1.0 hours (0.1 CEUs) of credit. See page 66 for learning objectives and test questions.

Copyright $\odot 1996$, Academy of Managed Care Pharmacy, Inc. All rights reserved 
search has emerged to define this value and demonstrate what the health care dollar is buying. This goes beyond the traditional proof of safety and efficacy of the product by including the description of the product's potential economic impact on a health care system. In the 1995 Zitter report on Pharmaceutical Outcomes Activities in the pharmaceutical industry, the number of outcomes activities decreased slightly despite a doubling of staff dedicated to outcomes activities. This trend seems to indicate an increased focus on outcomes research and pharmacoeconomic activities in the industry. Another trend of interest is the substantial increase in partnerships between the pharmaceutical industry and provider organizations in conducting outcomes research. ${ }^{2}$ Pharmacoeconomics can often be of greatest interest to the pharmaceutical industry because it provides valuable information on which products to develop.

\section{TERMINOLOGY}

The terms outcomes research and pharmacoeconomics must be distinguished from each other and various related words. The assessment of outcomes has been labeled outcomes research and is essentially the total quality management of health care.

Two key components of outcomes research include outcomes management and outcomes measurement. Outcomes management addresses the technology of measuring patient experience, while outcomes measurement defines the units for quantifying these outcomes. The process of health care delivery is tracked over time and the impact of particular health care choices on the patient's life is measured. Primary outcomes include medical resources used, cost of these resources, and quality of life.

A principal driver of outcomes research is the wide variation in practice patterns across the United States. Acute myocardial infarction may be treated differently at two different clinics within a managed care plan. The challenge of outcomes research is to determine which approach is most cost-effective, provides the best patient outcome, and find ways to have the same approach used in both areas.

Pharmacoeconomics is the most specific term in this arena. Pharmacoeconomics identifies, measures, and compares the costs and outcomes of using pharmaceutical products as a treatment alternatives. The most important objective of pharmacoeconomics is to demonstrate the value of using a pharmaceutical product in treating a specific disease. That value is often much greater than the price of a drug may indicate. For example, if taking a pharmaceutical appropriately for one year prevents a hospitalization, the overall cost for treating a patient is decreased because the cost of the hospitalization would far outweigh the annual cost of drug therapy. An additional example would include drugs dosed multiple times per day versus once a day, in which additional pharmacy preparation and nursing administration time and decreased compliance would involve additional costs. The side effect profile of different therapies can also have an impact on overall costs. Some side effects may be easily managed with inexpensive medication, when other side effects may require expensive concomitant medication, complicate co-morbid conditions, or require additional treatment. Drug formulation may be an issue if a patient needs to extend a hospital stay because the drug is only available intravenously.

When a therapy is over-used in populations where only a small group of patients will benefit, the average care cost per patient increases. On the other hand, if the population of patients can be identified where drug therapy will provide optimum outcomes, the cost per successful treatment can be substantially decreased.

Pharmacoeconomics is the science of establishing this value for pharmaceutical products.

\section{SOURCES AND USERS OF PHARMACOECONOMIC DATA}

Various health care systems are settings in which to conduct pharmacoeconomic research as well as users of pharmacoeconomic information.

Hospitals The hospital setting can provide excellent information on the care and cost of inpatient treatment. Hospitals also use pharmacoeconomic information to determine the impact of drug therapy on the length of stay for patients, as well as additional procedures that may be eliminated because of a particular drug therapy. In an institutional setting a pharmacist in charge of a drug budget may be reluctant to accept an expensive pharmaceutical product on the formulary. However, if pharmacoeconomic data can be presented to the pharmacist and/or the hospital administrator within the context of the overall cost perspective for the institution, the dimensions of decision making can be broadened. When used appropriately, pharmaceuticals can provide improved outcomes at equal or lower overall costs to the institution than current alternative therapy. As a hospital becomes part of an integrated health care delivery system, the pharmacoeconomic perspectives with which its personnel look at costs changes.

Managed care organizations Managed care systems can use pharmacoeconomic information since they are responsible for all aspects of care. They can also be a resource for pharmacoeconomic research through access to patient databases from either encounter data (staff or group model) or claims data (independent provider associations, preferred provider organizations, network models). When managed care contracts out to individual providers of care (i.e., physician groups, independent physician associations, hospitals, pharmacies, and long-term care facilities), attempts at conservation of costs are less coordinated among these individual groups. The managed care company reimburses each provider individually. However, in a staff or group model all providers of care take a personal and financial interest in keeping down the overall costs of health care delivery within their system. Therefore, the impact of pharmaceutical therapy on the various stages of an in- 
Table 1. Definitions of the Four Classic Pharmacoeconomic Models

Cost-effectiveness. An analysis that compares two or more interventions in terms of both total resources used and health outcomes achieved. Outcomes may be described as costs, charges, years of life saved, or days of hospitalization prevented.

Cost-utility. A type of cost-effectiveness analysis that evaluates costs and consequences of an intervention in terms of the patient's quality of life, ability to absorb cost, or preference for one treatment over another. Expressed as cost per quality-adjusted life-year (QALY).

Cost-minimization. An analysis of two or more treatments deemed to be comparably effective so that cost is the only differentiating factor. Also known as cost-identification analysis, cost-minimization compares direct medical costs.

Cost-benefit. In cost-benefit analysis, all costs (inputs) and benefits (consequences) of alternatives are measured in monetary terms. The outcome may be expressed as a ratio (benefit to cost) or in terms of net cost or benefit (benefit minus cost).

dividual's overall care are more relevant.

Pharmacy benefit management companies PBMs have traditionally focused on managing pharmaceutical costs for their managed care or employer clients. However, they are rapidly moving towards using pharmacoeconomic data in making formulary decisions that will produce the best overall economic outcome for their clients. This phenomenon has been recently described by Blissenbach as a move from determining total drug costs to calculating total medical costs. ${ }^{3}$ These two concepts are then combined to arrive at the "value equation" where value equals outcomes over costs.

As this trend emerges, PBMs will be able to use pharmacoeconomic data to justify an increase in per-member, permonth fees for the drug capitation that will result in further decreased medical costs. In the absence of these types of analyses, the PBM will no longer be able to remain competitive as a carved-out benefit

PBMs can also provide pharmaceutical claims information for the conduct of outcomes and pharmacoeconomic studies. As these organizations begin to work more closely with their managed care clients, they will also be in a position to offer integrated databases that incorporate the medical claims of their clients.

Integrated health care delivery systems As different providers join together to offer a continuum of care for patients, pharmacoeconomic information becomes of greater value. These systems are also in the process of creating information networks that incorporate data from each of the different provider areas. These data networks will be useful for conducting outcomes research projects. As these systems become more integrated, the emphasis on demonstrating the value of pharmaceutical therapy will be redirected toward the impact on overall health care costs.

Government Medicare and Medicaid programs are exploring various options for providing managed care and are becoming interested in data on the outcomes and usage of drug therapy. Pharmaceuticals are currently not covered for the elderly un-
Figure 1. Gaining Input from Different Countries Requires Various Methods of Communication to Achieve the Optimal Design in Pharmacoeconomic Studies.

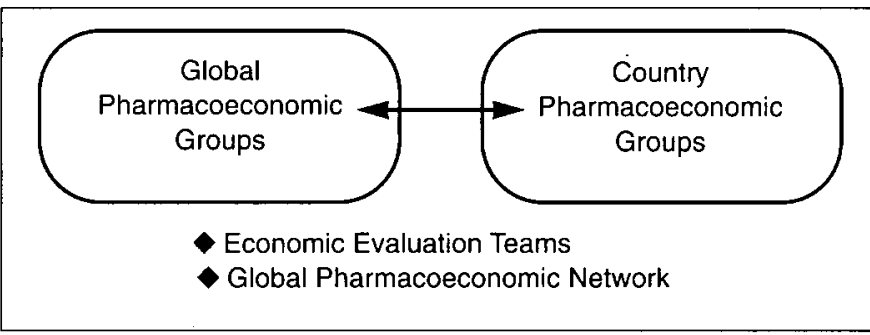

der Medicare. However, if patients are moved into a managed care program, a key inducement will be prescription drug coverage. These dynamics should provide valid data on the impact of prescription coverage on overall treatment costs for the elderly.

Medicaid programs can provide access to claims data for both medical and pharmacy services. These databases are an excellent resource for conducting retrospective analyses. However, the impact of pharmacoeconomic information is less pronounced in government-based care. Despite the agreement in principle of managing overall health care costs, most governments operate on an annual budget that promotes a more short-term outlook on costs, leading to the compartmentalization of budgets.

Food and Drug Administration The FDA focuses primarily on the aspect of promotion of pharmacoeconomic information. Currently, the FDA has no regulations for the design of pharmacoeconomic studies for promotion; however, the agency recommends that such evaluations be based on two randomized, well-controlled clinical trials. These recommendations differ significantly from the pharmacoeconomic needs of managed care customers and make FDA a unique customer for pharmacoeconomic information intended for promotional use.

In general, the design of a pharmacoeconomic study depends on the desired perspective customer group. A recent article by Draugalis and Coons ${ }^{4}$ provided a report of a conference that explored the facilitation of pharmacoeconomic research between academia, managed care organizations (MCOs), and the pharmaceutical industry. One of the key learnings from this conference was that the challenge to the pharmaceutical industry is to design studies sensitive to how the results will be accepted and used by the MCO. An even greater challenge will be to anticipate the needs of emerging customers such as employers and business coalitions, either directly or through the MCO

\section{CUSTOMIZED PHARMACOECONOMIC MODELS}

The classic terminology used in describing pharmacoeconomics includes the four models described in Table 1. Although these models provide the foundation of pharmacoeconomics, any one individual model often does not 
Table 2. Primary Objectives of U.S. Pharmaceutical Outcomes Research Group

$\Delta$ Assist in the design of worldwide pharmacoeconomic studies.

$\Delta$ Design pharmacoeconomic and outcomes research studies to support the U.S. market.

$\Delta$ Establish collaborative relationships with academia and the managed care industry in the area of outcomes research.

meet the specific needs of the customers of pharmacoeconomic data. Although the traditional models are critical in the design of pharmacoeconomic projects, the following definitions are a more practical description of the types of research projects often undertaken.

Cost of care evaluations provide models for the current cost of providing care for a particular disease state. They examine the natural history of disease and the medical resources and costs associated with treating that disease in a specific practice setting. An important distinction between a cost of care versus a cost of illness (COI) evaluation is the fact that a $\mathrm{COI}$ study will often be conducted from a societal perspective and include associated indirect costs secondary to the disease. In a cost of care model, the perspective is more often that of the payer and, therefore, indirect costs are of less interest. These types of projects provide information of benefit to both the MCO and the pharmaceutical industry. The MCO gains a detailed perspective on how a certain disease is managed within the plan, and the pharmaceutical industry obtains information on the potential economic impact of its product.

Phase III economic trials are those that collect data on economic parameters of drug therapy from the patients participating in Phase III clinical trials. This provides valuable economic information about the drug at the time of launch. A disadvantage of this approach is that the economic parameters may be driven by the clinical trial protocol and therefore may not reflect "real world" use of the drug. Drummond 5 summarizes the specific methodologic issues that arise when integrating economic and clinical research such as design, collection of resources-use data, collection of outcomes data, and the interpretation and extrapolation of results.

Naturalistic prospective evaluations are designed to address the limitation of Phase III economic trials by prospectively collecting the economic parameters of a drug and its most relevant therapeutic alternative within a specific practice setting with minimal intervention. Although these studies can often provide valuable information towards therapeutic decisions, a disadvantage is that they can be time consuming and expensive.

Retrospective database analysis of prescription and medical claims within a specific practice setting can provide useful information in a short period of time at little expense. Information on the trends of prescribing patterns can be particularly helpful. A disadvantage is that claims may not be an accurate reflection of actual care received if claims are submitted to maximize reimbursement.

Decision analysis modeling is an approach that uses in- formation from epidemiologic studies, clinical trials, administrative claims and cost databases, and expert opinion to model current care and the impact of a specific new therapy. These models can be very useful in designing prospective economic trials as well as offering predictions on the impact of a pharmaceutical agent in the treatment of disease. Because it is not always possible to study all the effects of treatment with clinical trials, modeling techniques can be very useful in making therapeutic decisions. These principles were recently reviewed by Stergachis. ${ }^{6}$

\section{HOW IS PHARMACOECONOMIC INFORMATION USED?}

Once this.information has been gathered, the challenge is how to use the information. Several areas of clinical practice have incorporated pharmacoeconomic data in relative decision analyses.

Pharmacoeconomics clearly defines the overall economic impact of drug therapy within all medical areas and presents the overall financial impact on a health care system.

An economic analysis of a particular drug can be especial ly useful in formulary decision making. A more expensive drug may decrease costs in other medical areas and could, therefore, be considered for the formulary. Often a drug will be cost-effective within a specific patient population. This information can be incorporated into clinical practice policies or. guidelines that describe how the drug can be used most efficiently to treat a specific disease or symptoms.

With pharmacoeconomic information in hand, caregivers can identify the true value of a drug to a health care system. This information can be used in price negotiations to support a pharmaceutical price premium or discount within a specilic plan.

\section{OPERATIONAL PHARMACOECONOMICS WITHIN THE PHARMACEUTICAL INDUSTRY}

How all this information is integrated into a pharmacoeconomic department within the pharmaceutical industry varies as much as the different approaches to conducting these studies. Pharmacoeconomic or outcomes research departments can be under the wing of marketing, clinical development, a product strategy group, or various other locations. At Ciba Pharmaceuticals, the Department of Pharmaceutical Out comes Research is based in the Policy and Business Development function. This geography allows for interactions with various departments, such as clinical development and marketing, while maintaining a specific identity for the pharmacoeconomic function.

The three principal functions of the U.S. Pharmaceutical Outcomes Research group at Ciba are described in Table 2. A secondary issue is whether a pharmacoeconomic or outcomes research function is part of a global initiative or a country specific-function. Since so many pharmaceutical companies are 
moving toward global research and development initiatives, the incorporation of pharmacoeconomic parameters into clinical trial design must also be globalized. However, in the area of pharmacoeconomics, this can pose several serious challenges. As countries make reimbursement decisions they are also developing country-specific guidelines for the conduct of pharmacoeconomic research in support of their decisions. Not surprisingly, the guidelines differ between countries. Another difference involves the practice patterns of care and the currency in which reimbursement takes place.

Because of all these market variances, the pharmaceutical industry should be represented by a global pharmacoeconomic strategy in conjunction with a global clinical research plan. The customer markets of the United States, principally managed care organizations, play a major role in the design of global pharmacoeconomic studies before launch. Therefore, a principal responsibility of a U.S.-based outcomes and pharmacoeconomics group should be to serve as a liaison between the country market and the global pharmacoeoncomic function.

To serve this function as a liaison, two important strategies have emerged. First, the incorporation of managed care sites into global clinical and pharmacoeconomic trials will provide managed care with a comprehensive understanding of the safety and efficacy of a product, within the health plan environment. This will allow informed decisions to be made at the time the product becomes available based on the performance of the drug in a managed care population. From the patients' perspective, they will have access to appropriate new therapies more quickly. Second, managed care should become more involved in designing market-specific models to augment the results available from global pharmacoeconomic studies. This would include both development of "cost of care" models for specific disease states and models to evaluate where the drug will be best positioned for maximum cost-efficiency once available.

\section{CONCLUSION}

In the current health care environment, all pharmaceutical products must provide the most beneficial outcomes at the lowest cost to patients, health care systems, and society at large. As an aid in managing this responsibility, the practice of outcomes research and pharmacoeconomics has emerged. Studies are being designed to meet the specific needs of the various providers of health care when making therapeutic decisions that include drug therapy. Pharmacoeconomic departments within the industry need to be structured to maximize the opportunity of incorporating economic parameters into ongoing clinical trials while at the same time providing data of practical use to the customers. Armed with outcomes and pharmacoeconomic information, the pharmaceutical industry is far better situated to meet the challenge of integrating into current practice.

\section{A References}

1. Morrison I, Leitman R. Health care outlook's eight critical issues. Presentation at the $1995 \mathrm{Ci}$ ba-Gigy Corporation Strategic Workshop. Summit, NJ: 1995 Nov 7

2. 1995 Pharmaceutical Outcomes Activities Study. San Francisco, CA: The Zitter Group and Technology Assessment Group, 1995.

3. Blissenbach HF. Use of cost-consequence mod- els in managed care. Pharmacotherapy 1995; 15 : 595-61S.

4. Draugalis JR, Coons SJ. Pharmacoeconomic research-facilitating collaboration among academic institutions, managed care organizations, and the pharmaceutical industry: a conference report. Clin Ther 1995; 17: 89-108.

5. Drummond MF, Davies L. Economic analysis alongside clinical trials-revisiting the methodological issues. Int J Technol Assess Health Care 1991; 7: 561-73.

6. Stergachis A. Overview of cost-consequence modeling in outcomes research. Pharmacotherapy 1995; 15: 40S-42S. 